\title{
Comparative analysis of single-band Wilkinson Power Dividers
}

\author{
Shilpa Mohanan $\mathrm{K}^{1}$, Sukanya Kulkarni ${ }^{2}$ \\ ${ }^{12}$ (Electronics and Telecommunication, Sardar Patel Institute of Technology, Mumbai,India)
}

\begin{abstract}
In the field of microwave engineering and circuit design, the Wilkinson Power Divider is an important type of power divider circuit that can achieve isolation between the output ports while maintaining a matched condition on all ports. Using RFSim99, a simple RF circuit simulator, an idealized Wilkinson can be simulated to help determine realistic values for return loss, isolation and other such characteristics. Similarly, two other topologies of Wilkinson Power Dividers are simulated and discussed. A comparison of all the three above configurations is carried out for their relations with regards to size of the design and S-parameters.
\end{abstract}

Keywords: Wilkinson, power-divider, single band, Sparameters

\section{Introduction}

Power dividers play a prominent role in microwave circuits dividing the power between the two or more ports depending upon the layout of the system[1]. Power divider splits the signal for large power amplifiers which may combine at the output to form a high power signal as observed in any WIMAX system.[2] An ideal power divider should demonstrate qualities of being matched, reciprocal and lossless. These power dividers can be constructed using various types of transmission lines Power dividers play a prominent role in microwave circuits dividing the power between the two or more ports depending upon the layout of the system[1]. Power divider splits the signal for large power amplifiers which may combine at the output to form a high power signal as observed in any WIMAX system.[2] An ideal power divider has the qualities of matching, reciprocity and being lossless. These power dividers can be constructed using various types of transmission lines (i.e. waveguides, microstrip, or strip line) or using resistive networks. Wilkinson power divider is used for many $\mathrm{RF}$ applications because it provides a low level of loss and maintains a high level of isolation between the output ports.

The Wilkinson power divider, proposed by Ernest Wilkinson in 1960 provides isolation between the output ports, is capable of being matched at all ports, and becomes lossless when the output ports are matched. [3] The original design consists of transmission lines with resistors connected between the lines. The connecting resistors are used to produce no voltage drop across them, and consequently, dissipate no power .Another important feature of Wilkinson power divider is that the construction can be easily done using microstrip which provides researchers to explore further. Wilkinson power divider configurations with different components are reported to date. Most of them include resistors, inductors and capacitors along with the transmission line. With the advancement of technology and usage of electronic devices on large, the same devices need to be used for several frequency bands. Keeping in mind size-reduction and compactness of the design, many research has been carried out towards this line of research.[4]-[6]

Section II describes a conventional Wilkinson power divider and its features. Section III provides an insight of another configuration with lumped elements only. Section IV discusses a third configuration with low-pass equivalent structures. A comparison is carried out in Section V and the paper is concluded in the next section.

\section{Conventional Wilkinson Power Divider}

The original design as proposed by Ernest has the following configuration of a transmission line with absorption resistors as shown in Fig.1. The RFSim99 schematic is shown in Fig 1. For the design at $1.8 \mathrm{GHz}$, the substrate assumed was RT Duroid 5870 board from Rogers Corporation with relative permittivity and loss tangent as 2.33 and 0.0005 respectively. The substrate thickness is $1.575 \mathrm{~mm}$ and the thickness of the copper tape is $31.75 \mu \mathrm{m}$. The skin depth of the copper was calculated to be approximately $1.5 \mathrm{~m}$ at $1.8 \mathrm{GHz}$. The length and width of the transmission line are calculated using microstrip equations to obtain them as $30.23 \mathrm{~mm}$ and 4.64 $\mathrm{mm}$. The normalized impedance was taken to be $50 \Omega$. 

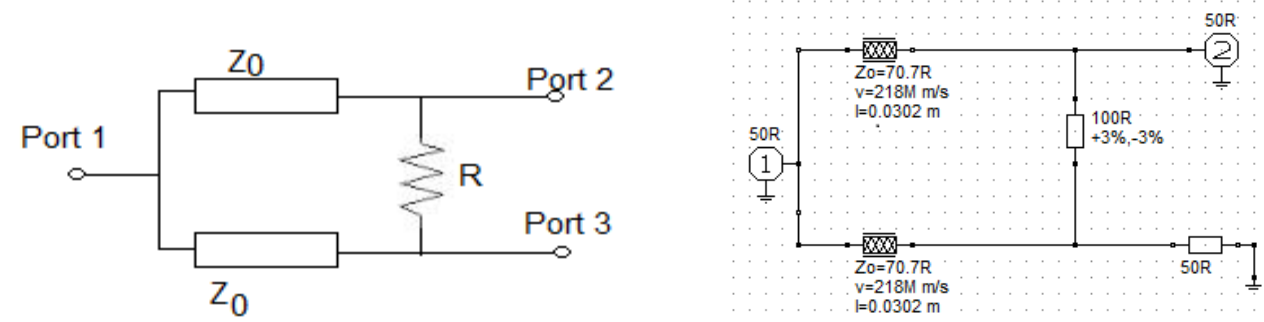

Fig. 1 a) Conventional Wilkinson Power Divider proposed by Ernest. b) RFSim 99 schematic of the design

The frequency responses of several S-parameters for a Wilkinson power divider were simulated in RFSim99 to determine the ideal performance. Fig. 2 gives the value for S11 and S12.Since the port for measurement was named as 1 and 2 for ports 2 and 3 respectively, we have S12 for S23 of the Wilkinson schematic. We observe the value of S23 to be around $-55.91 \mathrm{~dB}$ at the operating frequency. The graph in Fig. 3 shows that the results for S23 and S32 are essentially identical, further demonstrating the reciprocal nature of the Wilkinson. The frequency at which the isolation reaches a maximum value is approximately $1.8 \mathrm{GHz}$ equal to the operating frequency of the divider.

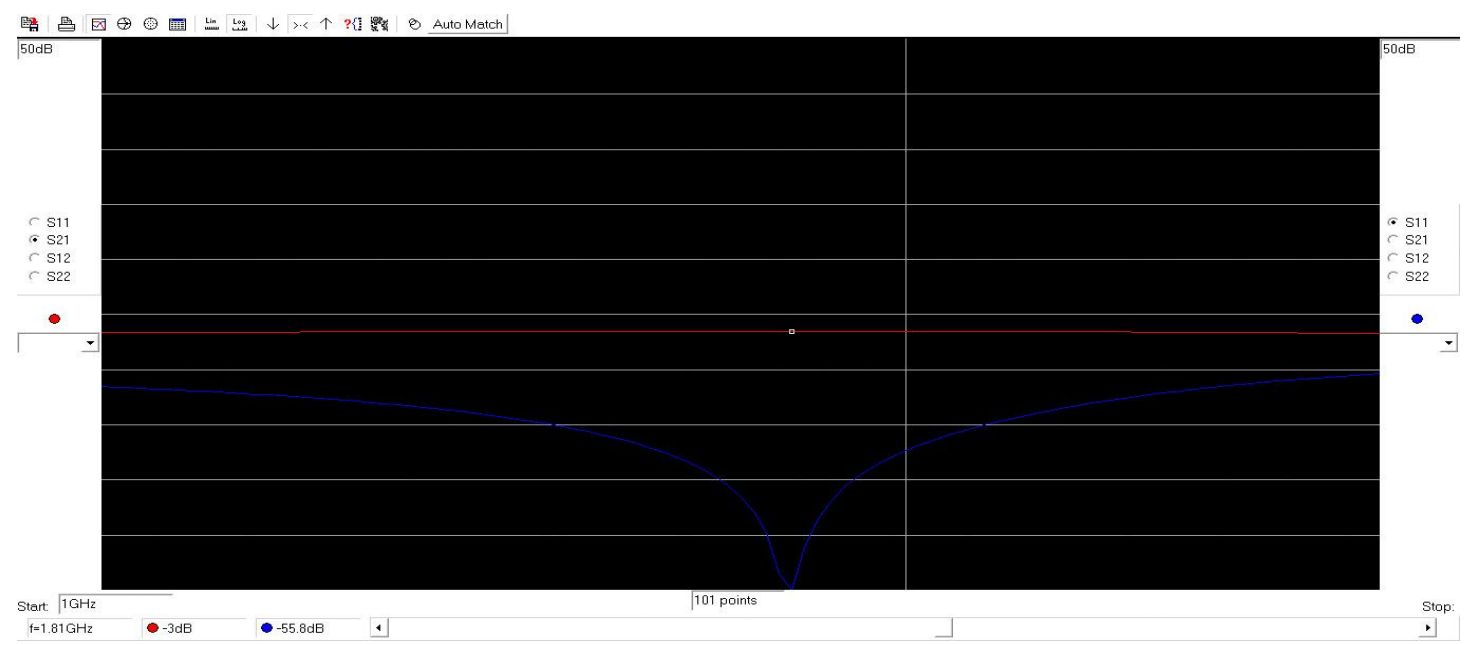

Fig. 2 S-parameter graph showing S11 and S12 for the conventional design

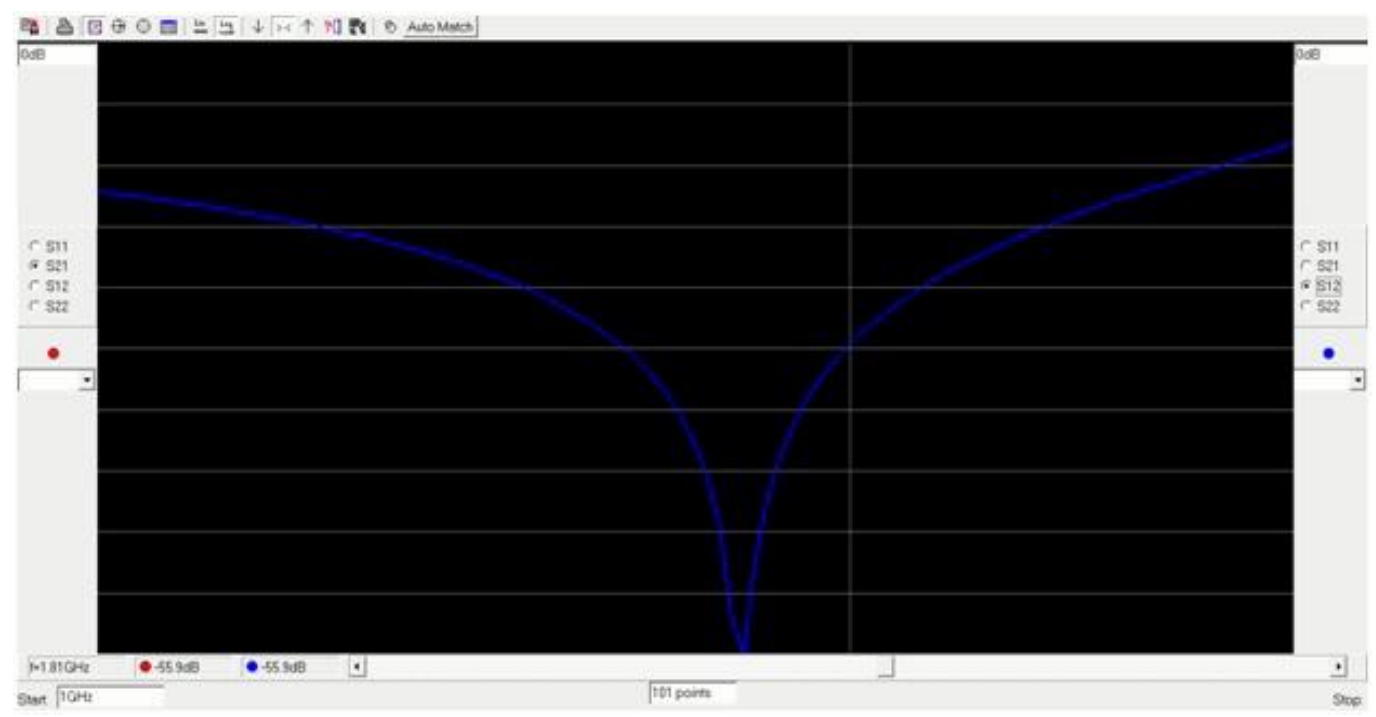

Fig. 3 S-parameter graph showing S23 and S32 for the original design 


\section{Wilkinson Power Divider Using Lumped-Elements}

The transmission line occupies a major portion of the design as per the configuration of Fig.1. To reduce the size of the overall design, lumped element equivalent of the transmission line is used to replace the quarter wave transmission line [7]. The RFSim99 schematic are shown in Fig. 4.The values for the elements are obtained as follows for $50 \Omega$ system impedance:

$$
\begin{array}{ll}
\mathrm{L}=11.25 / \mathrm{f}_{0}(\mathrm{nH}) & =6.25 \mathrm{nH} \\
\mathrm{C}=2.25 / \mathrm{f}_{0}(\mathrm{pF}) & =1.25 \mathrm{pF} \\
\mathrm{R}=100 \Omega & \\
\mathrm{f}_{0}=1 /(2 \pi \sqrt{ }(\mathrm{LC})) & =1.8 \mathrm{GHz}
\end{array}
$$

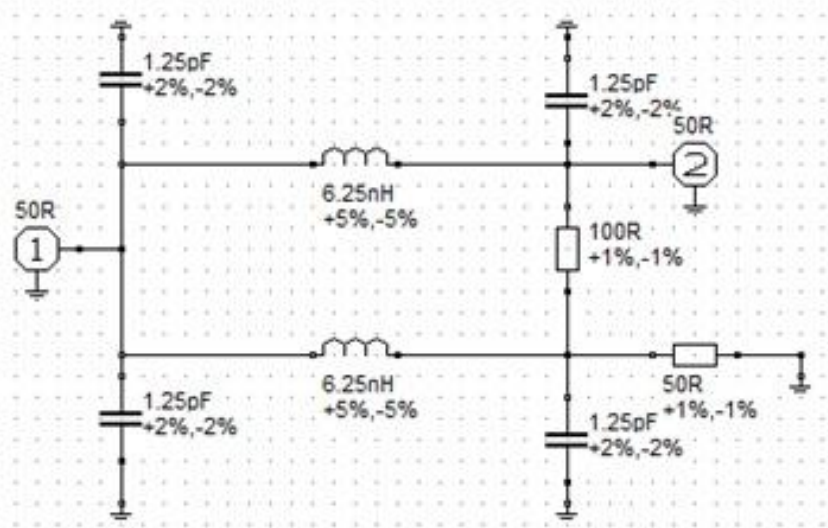

Fig. 4 RFSim99 schematic of lumped element design of the Wilkinson divider

The simulation shows the following results as seen in Fig. 5. The results show excellent graphs with $\mathrm{S} 11$ at maximum value of $-70.2 \mathrm{~dB}$ at $1.8 \mathrm{GHz}$ i.e. the operating frequency of the design. As required, the reflection loss is high whereas the transmission of around $-3 \mathrm{~dB}$ was obtained at frequency as shown by S12.Similarly, from the Fig 6, we can observe that the value of S23 would be $-50.5 \mathrm{~dB}$ near the frequency.

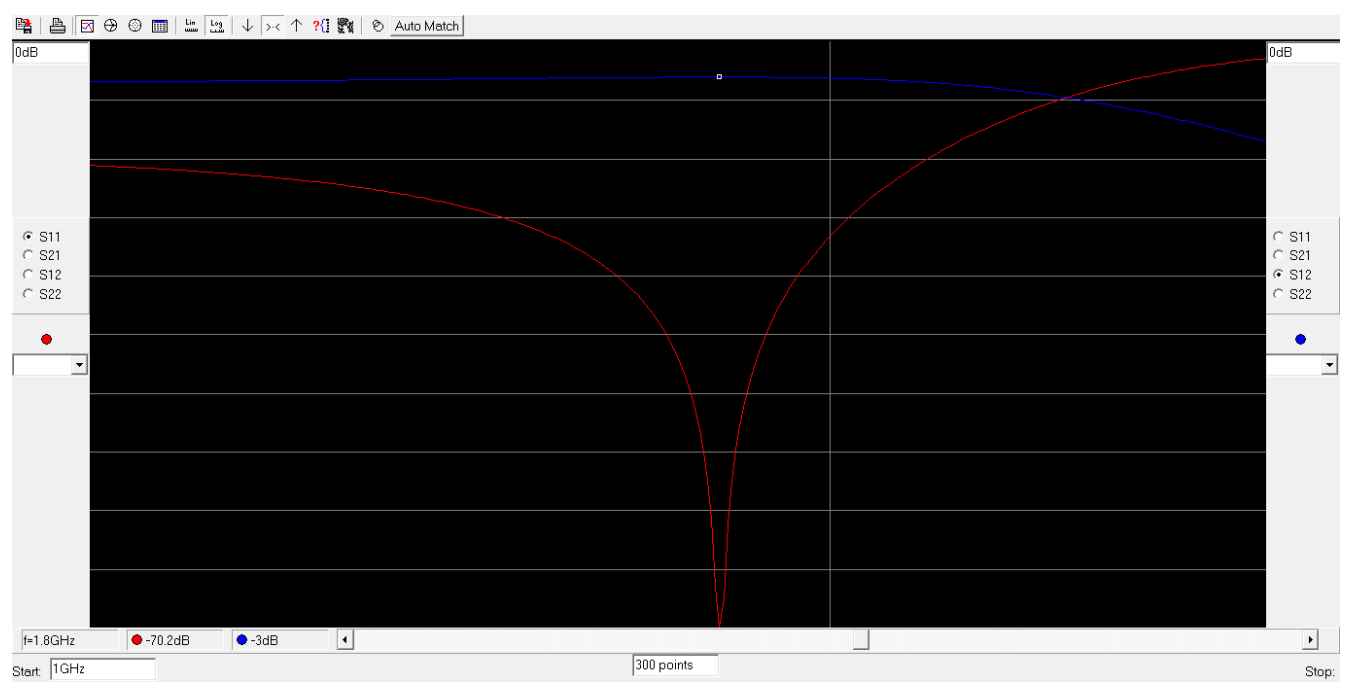

Fig 5. S-parameter graph showing S11 and S12 for lumped element design 


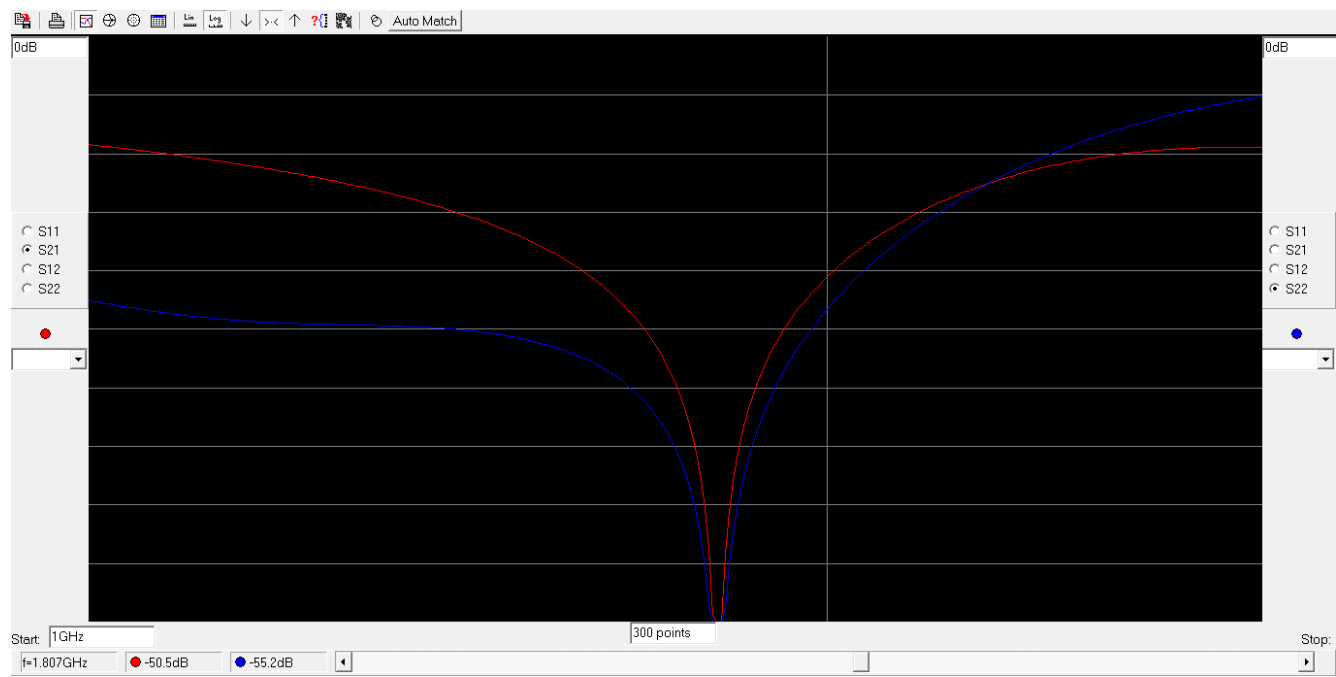

Fig. 6 S-parameter graph showing S23 and S33 for lumped element design

\section{Low-Pass Equivalent Wilkinson Power Divider}

The main element of any Wilkinson power divider is the quarter-wave transmission line. If these lines are replaced by their low-pass equivalent structures, then the similar approach can be obtained. For the low-pass structure, lambda/8 transmission line at the operating frequency and two capacitors are used to form a pinetwork.[7] The values for the capacitance can be obtained by comparing the low-pass structure with the quarter-wave transmission line. Thus, for particular characteristic impedance and an operating frequency, suitable capacitance value can be calculated as:

$$
\mathrm{C}=1 /\left(2 \pi \sqrt{ } 2 \mathrm{Z}_{0}\right)
$$

Using the addition of a shunt capacitance as previously described, a reduced size topology of the original Wilkinson divider is presented in Fig. 7(a).The schematic in RFSim99 is as shown in Fig. 7 (b) .

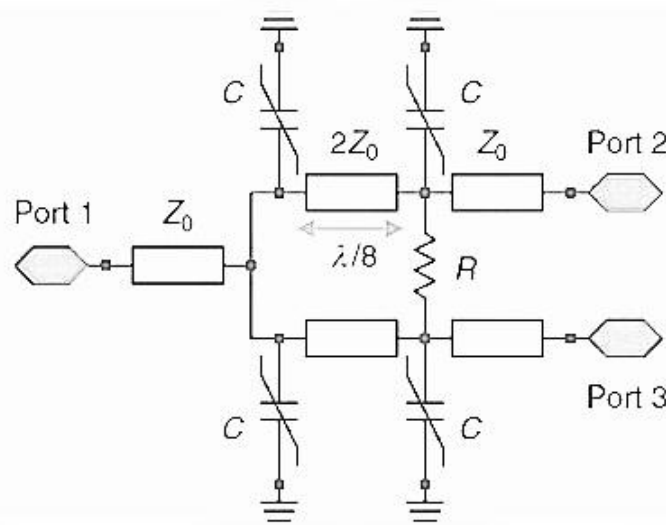

Fig 7. Low-pass equivalent design for Wilkinson power Divider a) Layout

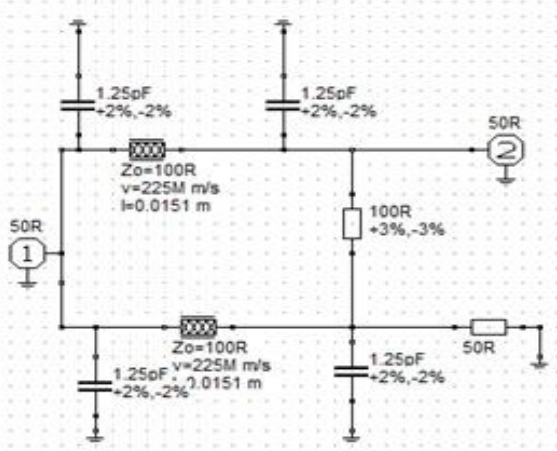

b) RFSim99 schematic.

Fig. 8 shows the transmitted power to port 2 from port 1 which lies around $3 \mathrm{~dB}$ in the frequency band of operation. The other parameter is the reflection loss shown by S11 which falls to 21.6dB. Fig. 9 gives the graph for isolation between ports 2 and 3 in the band of action. 


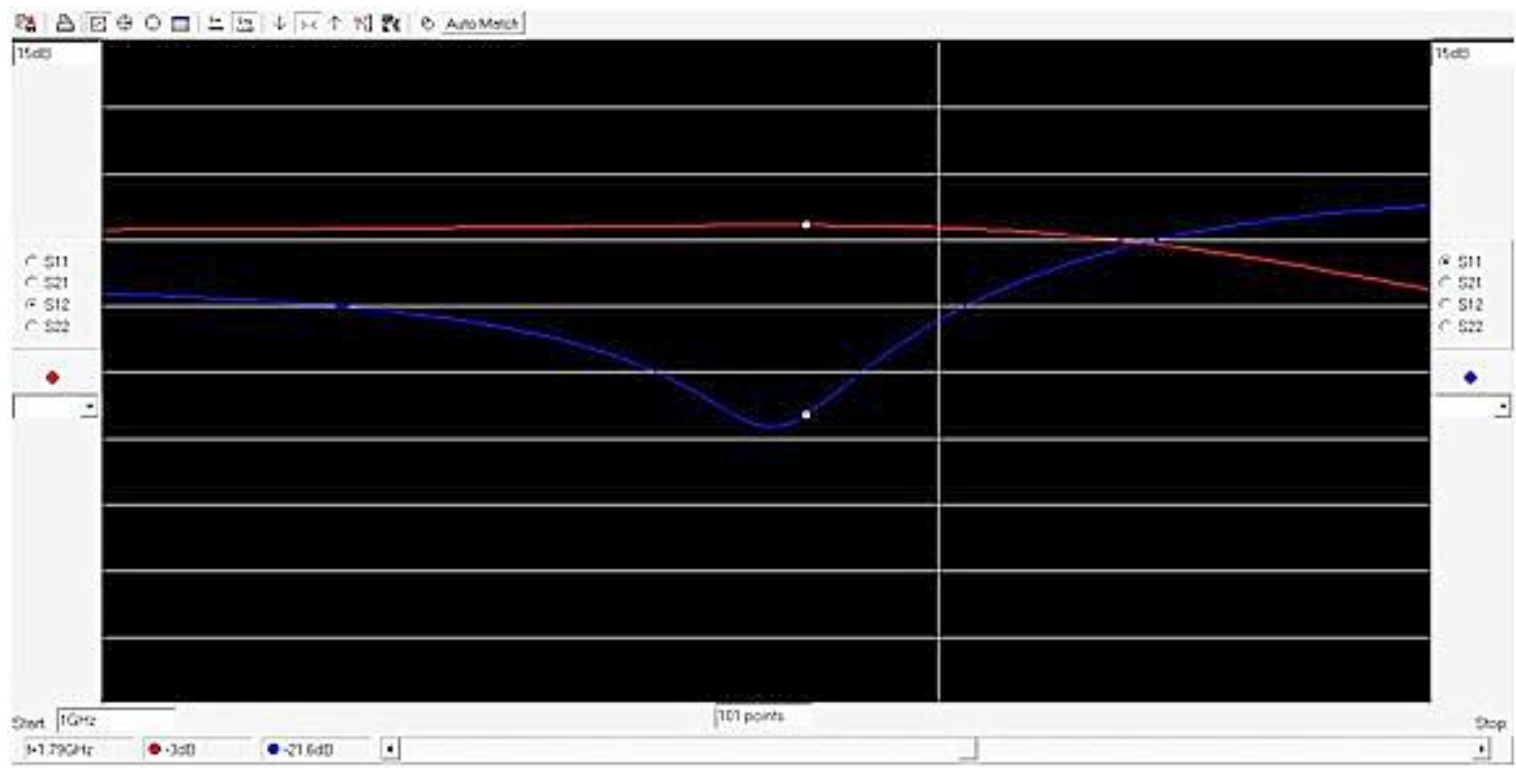

Fig. 8 S-parameter graph showing S12 and S11 for low-pass equivalent design

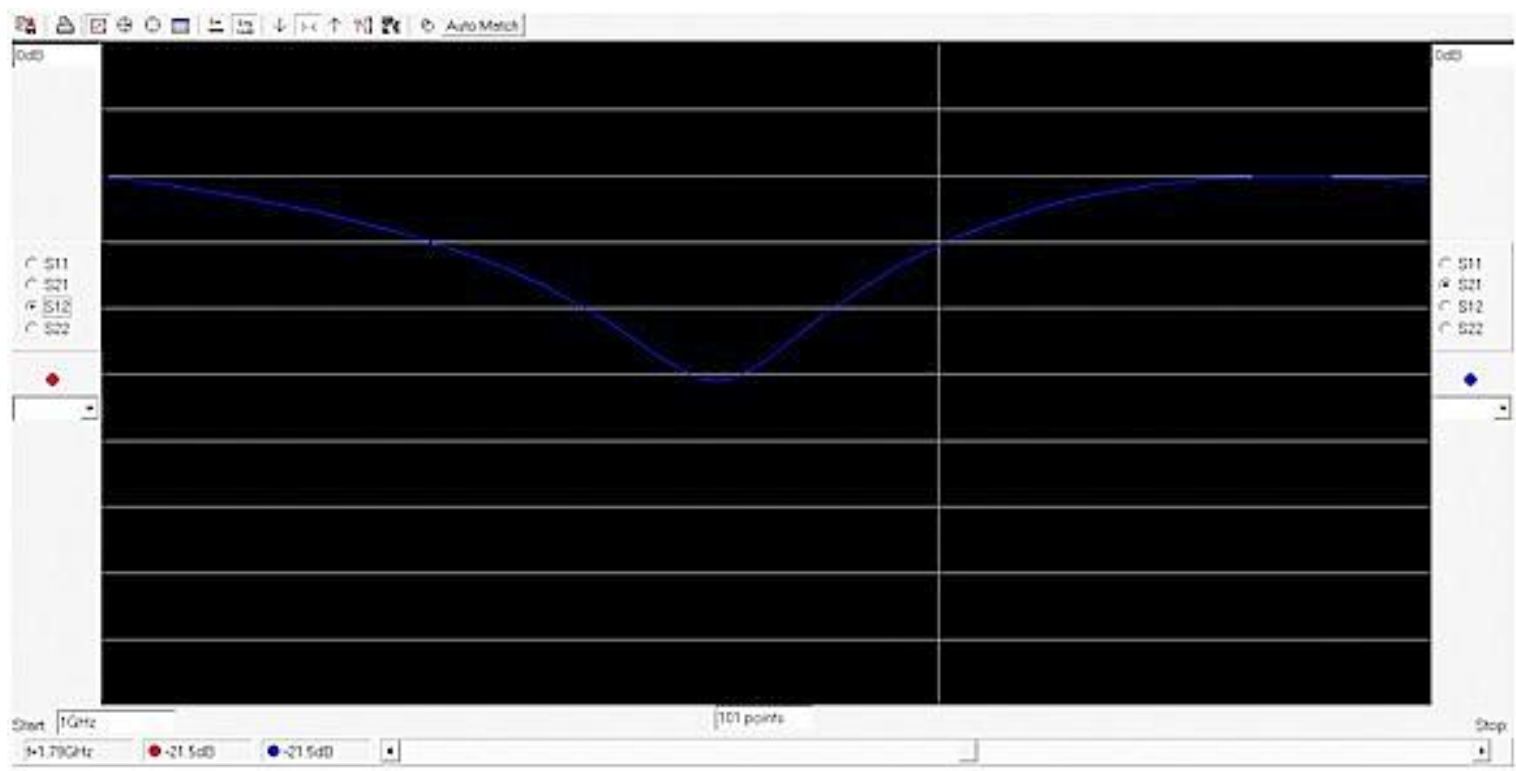

Fig. 9 S-parameter graph showing S23 for low-pass equivalent design

\section{Comparison Of The Three Configurations}

Table 1 shows the comparison of the three configurations. S-parameters in $\mathrm{dB}$ are compared for their characteristics of isolation, power-division, and reflection. Lumped element configuration shows a promising result when compared with the original design. But the only point for consideration is the bandwidth available. The bandwidth reduction of about $45-55 \%$ is shown in the lumped element design. The added feature for the lumped element design is the ease in fabrication.

On the other hand, the low-pass equivalent structure shows a considerable decrease in the length of the design providing adequate values for reflection and isolation in the band of operation.

Table 2 Comparisons Among The Three Designs Of Wilkinson Power Divider Showing The Parameters In Db

\begin{tabular}{|c|c|c|c|}
\hline Parameters & Original design & Lumped element design & Low-pass design \\
\hline S11/S22 & -55.8 & -70.2 & -21.6 \\
\hline S12/S21 & -3 & -3 & -3 \\
\hline S23/S32 & -55.91 & -50.5 & -21.5 \\
\hline
\end{tabular}




\section{Conclusion}

The three configurations are simulated and compared for their characteristics. The original design with quarter wave transmission line has the largest area when compared to the other two. Lumped element design shows ease of fabrication whereas the third design size reduction to the maximum. Thus, depending upon the requirement of minimum area or best reflection or isolation, the configuration can be wisely chosen.

\section{References}

[1] David M. Pozar, Microwave Engineering (John Wiley \& Sons Inc, New York, 2005).

[2] U.D. Dalal, Y. P. Kosta, WIMAX New Developments( Croatia: Intech 2009, ch. 10).

[3] E.J.Wilkinson, An N-way hybrid power divider, IRE Transactions Microwave Theory Techniques, vol MTT-8, no.1, Jan 1960, pp. 116-118

[4] X.Wang, I. Sakagami,K. Takahashi and S. Okamura, “A Generalized Dual-Band Wilkinson Power Divider With Parallel L,C and R Components, IEEE Transactions on Microwave Theory and Techniques, vol. 60, no. 4, pp. 952-964, April 2012.

[5] X. H. Wang, Y. F. Bai, H. Xu, Q. L. Huang, and X. W. Shi, "Dual-band Power Divider with High Frequency-ratio, Microwave and MillimeterWave Technology (ICMMT), vol. 2, pp. 1-4, May 2012.

[6] I. Sakagami, X. Wang, K. Takahashi, and S. Okamura, "Generalized Two-Way Two-Section Dual-Band Wilkinson Power divider With Two Absorption Resistors and Its Miniaturization, IEEE Transactions on Microwave Theory and Techniques, vol. 59, no. 11, pp. 2833-2847, Nov 2011.

[7] E. Lourandakis, R. Weigel,H. Mextorf and R. Knoechel, “Circuit Agility,” IEEE Microwave Magazine, pp.111-121,Jan 2012. 